\title{
BENCHMARKING JUDGMENTALLY ADJUSTED FORECASTS
}

\author{
PHILIP HANS FRANSES ${ }^{*, \dagger}$ and BERT DE BRUIJN \\ Econometric Institute, Erasmus School of Economics, Rotterdam, The Netherlands
}

\begin{abstract}
Many publicly available macroeconomic forecasts are judgmentally adjusted model-based forecasts. In practice, usually only a single final forecast is available, and not the underlying econometric model, nor are the size and reason for adjustment known. Hence, the relative weights given to the model forecasts and to the judgement are usually unknown to the analyst.

This paper proposes a methodology to evaluate the quality of such final forecasts, also to allow learning from past errors. To do so, the analyst needs benchmark forecasts. We propose two such benchmarks. The first is the simple no-change forecast, which is the bottom line forecast that an expert should be able to improve. The second benchmark is an estimated model-based forecast, which is found as the best forecast given the realizations and the final forecasts. We illustrate this methodology for two sets of GDP growth forecasts, one for the USA and one for the Netherlands. These applications tell us that adjustment appears most effective in periods of first recovery from a recession. Copyright (C) 2016 John Wiley \& Sons, Ltd.
\end{abstract}

Received 03 June 2016; Accepted 13 September 2016

JEL CODE: C20; C51

KEY WORDS: forecast decomposition; expert adjustment; total least squares

\section{INTRODUCTION}

Many publicly available macroeconomic forecasts are judgmentally adjusted model-based forecasts. Econometric models can be multiple-equation systems with hundreds of variables or identities, or Bayesian vector autoregressions or even simple extrapolation tools. An illustration of the first is given by Franses, Kranendonk and Lanser (2011), where all the forecasts from the large macroeconomic model of the Netherlands Bureau for Economic Policy Analysis (CPB) are manually adjusted by experts with domain-specific knowledge.

In many situations, it can be beneficial to adjust model-based forecasts. When experts foresee that a prediction error is to be made with the model, then adjustment can help to improve accuracy. For example, adjustment can be needed because of measurement issues in the explanatory variables at the forecast origin or because of anticipated changes, not included in the model at the forecast origin.

Despite the potential success of expert adjustment, it is rarely documented what an expert does and why certain decisions have been made. This hampers a straightforward evaluation of forecast errors, as it is usually unknown which part of the error could be due to the econometric model and which part to the manual adjustment. In other words, the relative weights given to the econometric model forecasts and to the judgement are usually unknown to the analyst.

In this paper, we propose a methodology that allows to study the merits of the relative contribution of an expert. In fact, our methodology allows to indicate when, that is, for which years or quarters, did the expert make the final forecast better than an underlying model forecast and when did the expert touch harm that forecast quality? For this

\footnotetext{
*Correspondence to: Philip Hans Franses, Econometric Institute, Erasmus School of Economics, Rotterdam, The Netherlands.

†E-mail: franses@ese.eur.nl

Thanks are due to Richard Paap, Christiaan Heij and Tom Wansbeek for various helpful comments.
} 
methodology, we need benchmark econometric model forecasts. Now, typically, one resorts to the simplest benchmark possible, and this is the no-change forecast, see Vuchelen and Gutierrez (2005) and also recently Franses and Maassen (2015). The idea is that an expert would not show much expertise if this trivial forecast cannot be beaten. In the present paper, we additionally propose another benchmark forecast, and this associates with in some sense a 'best model-based' forecast. We derive this best forecast from the final forecasts and the realizations, and use the technique called total least squares (TLS), which here in our setting of forecasts and realizations boils down to the so-called Deming regression (Deming, 1943). We illustrate our methodology using two sets of forecasts for growth in gross domestic product (GDP), one for the Netherlands and one for the USA. Zooming in on successful contributions of the experts, we find that they have in common that they have been particularly successful in the first periods of recovery from a recession as then the experts' added valuable information to the model forecast.

The outline of our paper is as follows. Section 2 introduces the two benchmark model-based forecasts, where most attention will be given to the 'best model-based' forecast. Section 3 presents a detailed illustration of our methodology, and Section 4 concludes.

\section{BENCHMARK MODEL-BASED FORECASTS}

When an analyst wants to evaluate the quality of forecasts, say from the IMF, OECD and the World Bank, or, as in our illustration later, wants to analyse the qualities of the Econometric Institute Current Indicator of the Economy (EICIE), then a benchmark is needed. In some situations, typically in business forecasting, there is the availability of the actual model-based forecasts, see Franses (2014) for a review, but in many other situations, typically in macroeconomics, such model-based forecasts are not available.

\subsection{The no-change forecast}

A first and simple benchmark forecast is of course the no-change forecast. That is, if we consider a variable $y_{t}$ that needs to be predicted, then the one-step-ahead no-change forecast is $y_{t-1}$.

Denoting the final expert-adjusted forecast as $f_{t}$, Vuchelen and Gutierrez (2005) advocate the use of this no-change forecast in their auxiliary regression:

$$
y_{t}=\mu+\gamma_{1} y_{t-1}+\gamma_{2}\left(f_{t}-y_{t-1}\right)+\varepsilon_{t}
$$

where they advocate a Wald test for the composite null hypothesis that $\mu=0, \gamma_{1}=1, \gamma_{2}=1$. Under this null hypothesis, the model-based forecast is unbiased, and the expert adjustment on top of that no-change forecast is then unbiased, too. If the null hypothesis is rejected, one can have a closer look at the estimated parameter values of $\gamma_{1}$ and $\gamma_{2}$.

\subsection{The best model-based forecast}

To arrive at a method to retrieve an estimator of the 'best model-based' forecast, we somehow need to make assumptions. A first assumption is that an observed expert-adjusted forecast $f_{t}$ is a forecast of a variable $y_{t}^{*}$, which is the true variable of interest, but that this true variable is measured with error, hence $y_{t}$. Next, we assume that $f_{t}$ amounts to a concerted outcome of an econometric model forecast $f_{t}^{M}$ and an expert touch $f_{t}^{E}$, with

$$
f_{t}=f_{t}^{M}+f_{t}^{E}
$$

The third assumption is that $f_{t}^{E}$ and $f_{t}^{M}$ are independent. This assumption corresponds with an optimal situation, as when it does not hold, the expert is adding something to the model forecast that is already in there, and this amounts to double counting.

Our simple method to estimate $f_{t}^{M}$ and $f_{t}^{E}$ from $f_{t}$ and the realizations $y_{t}$ relies on the familiar regression:

$$
y_{t}^{*}=\alpha+\beta f_{t}^{M}+\varepsilon_{t}
$$


which is usually used to test if $\alpha=0$ and $\beta=1$, where these parameter values associate with unbiased forecasts. Our method is now based on the assumption that the two variables in Equation (3) are measured with error. First, as mentioned, for $y_{t}^{*}$, we assume that

$$
y_{t}=y_{t}^{*}+w_{t}
$$

where $w_{t}$ has variance $\sigma_{w}^{2}$ and $w_{t}$ is independent from $y_{t}^{*}$ and the $\varepsilon_{t}$ in Equation (3). For $f_{t}^{M}$, we introduce a measurement error via Equation (2), that is, $f_{t}=f_{t}^{M}+f_{t}^{E}$, which thus treats the expert touch as a measurement error. The $f_{t}^{E}$ has variance $\sigma_{E}^{2}, f_{t}^{M}$ has variance $\sigma_{M}^{2}$, and as said, we further assume that $f_{t}^{E}$ and $f_{t}^{M}$ are independent, so the variance of $f_{t}$ is $\sigma_{F}^{2}=\sigma_{M}^{2}+\sigma_{E}^{2}$.

For practical purposes, it is interesting to estimate $f_{t}^{E}$ and $f_{t}^{M}$, and in particular the variances $\sigma_{E}^{2}$ and $\sigma_{M}^{2}$. It is also important to study the model-based forecast errors $y_{t}-f_{t}^{M}$ versus $y_{t}-f_{t}$ to learn about the contribution of the expert. That is, does the expert touch lead to better forecasts?

In sum, the key unobserved variable to estimate is $f_{t}^{M}$ using data on $y_{t}$ and $f_{t}$. We now propose a methodology to do so. The key problem that we face is estimating $f_{t}^{M}$, given that the true regression model is $y_{t}^{*}=\alpha+\beta f_{t}^{M}+\varepsilon_{t}$ and that the data are assumed to follow from $y_{t}=y_{t}^{*}+w_{t}$ and $f_{t}=f_{t}^{M}+f_{t}^{E}$, which is the case of measurement errors in two variables, the dependent and the independent variables. There are many techniques available that usually focus on obtaining consistent estimators of $\alpha$ and $\beta$, see, for example, Koopmans (1937), Fuller (1987) and Wansbeek and Meijer (2000). One technique, which goes back to Frisch (1933), is particularly useful as it delivers a simple estimator to predict the values of $f_{t}^{M}$. This method is called TLS, and it is also sometimes coined as the Deming regression (Deming, 1943).

An alternative least squares estimator for $\beta$ is the TLS estimator, which seeks to minimize the squares of the orthogonal distances to the regression line. It is thus assumed that part of the error in the regression model corresponds with a measurement error in the dependent variable. Define

$$
\delta=\frac{\sigma_{\varepsilon}^{2}+\sigma_{w}^{2}}{\sigma_{E}^{2}}
$$

see Carroll and Ruppert (1996), and define $\bar{y}=\frac{1}{T} \sum_{t=1}^{T} y_{t}$ and $\bar{f}=\frac{1}{T} \sum_{t=1}^{T} f_{t}$, where $T$ is the number of one-step-ahead forecasts. The TLS estimators for $\beta$ and $\alpha$, now converge to

$$
\begin{gathered}
\hat{\beta}_{T L S} \rightarrow \frac{\sigma_{y}^{2}-\delta \sigma_{F}^{2}+\sqrt{\left(\sigma_{y}^{2}-\delta \sigma_{F}^{2}\right)^{2}+4 \delta \sigma_{F y}^{2}}}{2 \sigma_{F y}} \\
\hat{\alpha}_{T L S} \rightarrow \bar{y}-\hat{\beta}_{T L S} \bar{f}
\end{gathered}
$$

Table 1. Average correlation between the predicted measurement-error-free explanatory variable and its true observations, and the percentage unexplained of the true observations

\begin{tabular}{lccc}
\hline & $T$ & Correlation & Percentage unexplained \\
\hline$\sigma_{w}^{2}=1, \sigma_{v}^{2}=1, \sigma_{\varepsilon}^{2}=0, \sigma_{x^{*}}^{2}=1$ & 100 & 0.912 & 22.4 \\
$\sigma_{w}^{2}=1, \sigma_{v}^{2}=1, \sigma_{\varepsilon}^{2}=\mathbf{1} \sigma_{x^{*}}^{2}=1$ & 500 & 0.913 & 20.5 \\
$\sigma_{w}^{2}=1, \sigma_{v}^{2}=1, \sigma_{\varepsilon}^{2}=\mathbf{2}, \sigma_{x^{*}}^{2}=1$ & 100 & 0.864 & 36.2 \\
$\sigma_{w}^{2}=\mathbf{2}, \sigma_{v}^{2}=1, \sigma_{\varepsilon}^{2}=0, \sigma_{x^{*}}^{2}=1$ & 500 & 0.866 & 33.8 \\
$\sigma_{w}^{2}=1, \sigma_{v}^{2}=\mathbf{2}, \sigma_{\varepsilon}^{2}=0, \sigma_{x^{*}}^{2}=1$ & 100 & 0.798 & 58.8 \\
$\sigma_{w}^{2}=1, \sigma_{v}^{2}=1, \sigma_{\varepsilon}^{2}=0, \sigma_{x^{*}}^{2}=\mathbf{2}$ & 500 & 0.801 & 56.1 \\
& 100 & 0.813 & 50.9 \\
& 500 & 0.816 & 22.9 \\
& 100 & 0.898 & 25.6 \\
& 500 & 0.899 & 5.5 \\
\hline
\end{tabular}

The setting is $y_{t}=y_{t}^{*}+w_{t}$, with $\sigma_{t}^{2}=x_{t}^{*}+v_{t}$, with $\sigma_{v}^{2}$ Data Generating Process (DGP): $y_{t}^{*}=-1+2 x_{t}^{*}+\varepsilon_{t}$, with $\sigma_{\varepsilon}^{2}$ where $y_{t}^{*}, w_{t}, x_{t}^{*}, v_{t}, \varepsilon_{t}$ are draws from an $\mathrm{N}(0,1)$ distribution. Simulations are for samples $T=100$ and 500, and the number of replications is 10000 . It is assumed that $\delta$ is known. 
PHILIP HANS FRANSES AND BERT DE BRUIJN

Table 2. Average correlation between the predicted measurement-error-free explanatory variable and its true observations, and the percentage unexplained of the true observations

\begin{tabular}{lccc}
\hline & $T$ & Correlation & Percentage unexplained \\
\hline$\sigma_{w}^{2}=1, \sigma_{v}^{2}=1, \sigma_{\varepsilon}^{2}=0, \sigma_{x^{*}}^{2}=1$ & 100 & 0.907 & 20.6 \\
$\sigma_{w}^{2}=1, \sigma_{v}^{2}=1, \sigma_{\varepsilon}^{2}=\mathbf{1} \sigma_{x^{*}}^{2}=1$ & 500 & 0.908 & 18.8 \\
$\sigma_{w}^{2}=1, \sigma_{v}^{2}=1, \sigma_{\varepsilon}^{2}=\mathbf{2}, \sigma_{x^{*}}^{2}=1$ & 100 & 0.852 & 31.6 \\
$\sigma_{w}^{2}=\mathbf{2}, \sigma_{v}^{2}=1, \sigma_{\varepsilon}^{2}=0, \sigma_{x^{*}}^{2}=1$ & 500 & 0.853 & 29.8 \\
$\sigma_{w}^{2}=1, \sigma_{v}^{2}=\mathbf{2}, \sigma_{\varepsilon}^{2}=0, \sigma_{x^{*}}^{2}=1$ & 100 & 0.765 & 48.9 \\
$\sigma_{w}^{2}=1, \sigma_{v}^{2}=1, \sigma_{\varepsilon}^{2}=0, \sigma_{x^{*}}^{2}=\mathbf{2}$ & 500 & 0.767 & 47.0 \\
& 100 & 0.786 & 44.7 \\
& 500 & 0.788 & 42.8 \\
& 100 & 0.897 & 29.4 \\
& 500 & 0.898 & 22.1 \\
\hline
\end{tabular}

The setting is $y_{t}=y_{t}^{*}+w_{t}$, with $\sigma_{t}^{2}=x_{t}^{*}+v_{t}$, with $\sigma_{v}^{2}$ Data Generating Process (DGP): $y_{t}^{*}=-1+2 x_{t}^{*}+\varepsilon_{t}$, with $\sigma_{\varepsilon}^{2}$ where $y_{t}^{*}$, $w_{t}, x_{t}^{*}, v_{t}, \varepsilon_{t}$ are draws from an $\mathrm{N}(0,1)$ distribution. Simulations are for samples $T=100$ and 500, and the number of replications is 10000 . It is assumed that $\delta$ is incorrectly specified as $\frac{1}{2} \boldsymbol{\delta}$.

where we denote as $\sigma_{F y}$ the covariance between the observed series and its forecasts, see Deming (1943, page 184). In practice, these TLS estimators are of course based on the sample equivalents of the variances and covariance. The key feature of this method, which is relevant for our purposes, is that an interesting by-product of TLS is an estimator for the measurement-error-free explanatory variable, that is,

$$
\hat{f}_{t}^{M}=f_{t}+\frac{\hat{\beta}_{T L S}}{\hat{\beta}_{T L S}^{2}+\delta}\left(y_{t}-\hat{\alpha}_{T L S}-\hat{\beta}_{T L S} f_{t}\right)
$$

see Linnet (1990). Our key assumption now is that we will coin this $f_{t}^{M}$ as the 'best model-based' forecast in our illustrations later.

The key parameter that one should set from the outset is $\delta$ in Equation (5). Given our particular case of realizations and forecasts, it may not be unreasonable to assume that $\sigma_{w}^{2}=\sigma_{E}^{2}$. Then

$$
\delta=\frac{\sigma_{\varepsilon}^{2}}{\sigma_{E}^{2}}+1
$$

Table 3. Average correlation between the predicted measurement-error-free explanatory variable and its true observations, and the percentage unexplained of the true observations

\begin{tabular}{lccc}
\hline & $T$ & Correlation & Percentage unexplained \\
\hline$\sigma_{w}^{2}=1, \sigma_{v}^{2}=1, \sigma_{\varepsilon}^{2}=0, \sigma_{x^{*}}^{2}=1$ & 100 & 0.898 & 33.2 \\
$\sigma_{w}^{2}=1, \sigma_{v}^{2}=1, \sigma_{\varepsilon}^{2}=\mathbf{1} \sigma_{x^{*}}^{2}=1$ & 500 & 0.899 & 30.9 \\
$\sigma_{w}^{2}=1, \sigma_{v}^{2}=1, \sigma_{\varepsilon}^{2}=\mathbf{2}, \sigma_{x^{*}}^{2}=1$ & 100 & 0.840 & 54.1 \\
$\sigma_{w}^{2}=\mathbf{2}, \sigma_{v}^{2}=1, \sigma_{\varepsilon}^{2}=0, \sigma_{x^{*}}^{2}=1$ & 500 & 0.842 & 52.0 \\
$\sigma_{w}^{2}=1, \sigma_{v}^{2}=\mathbf{2}, \sigma_{\varepsilon}^{2}=0, \sigma_{x^{*}}^{2}=1$ & 100 & 0.772 & 78.8 \\
$\sigma_{w}^{2}=1, \sigma_{v}^{2}=1, \sigma_{\varepsilon}^{2}=0, \sigma_{x^{*}}^{2}=\mathbf{2}$ & 500 & 0.774 & 76.7 \\
& 100 & 0.787 & 73.8 \\
& 500 & 0.788 & 71.6 \\
& 100 & 0.888 & 58.5 \\
\end{tabular}

The setting is $y_{t}=y_{t}^{*}+w_{t}$, with $\sigma_{t^{*}}^{2}=x_{t}^{*}+v_{t}$, with $\sigma_{v}^{2}$ Data Generating Process (DGP): $y_{t}^{*}=-1+2 x_{t}^{*}+\varepsilon_{t}$, with $\sigma_{\varepsilon}^{2}$ where $y_{t}^{*}, w_{t}, x_{t}^{*}, v_{t}, \varepsilon_{t}$ are draws from an $\mathrm{N}(0,1)$ distribution. Simulations are for samples $T=100$ and 500 , and the number of replications is 10000 . It is assumed that $\delta$ is incorrectly specified as $2 \boldsymbol{\delta}$. 
Simulation results in Table 1 show that, in case the value of $\delta$ is known, the correlation between simulated $f_{t}^{M}$ and estimated $f_{t}^{M}$ ranges from around 0.8 to close to 1 . The size of the unexplained part depends on the variances and can range from $5 \%$ to close to $60 \%$. The sample size does not seem to matter. Tables 2 and 3 present the results for the cases where the true value of $\delta$ is deliberately underestimated by a fraction $\frac{1}{2}$ and deliberately overestimated by a fraction 2, respectively. In general, the correlations do not differ much from those in Table 1. For the explained part, we see that overestimation leads to a slightly larger fraction of the unexplained part.

In the next section, we apply our methodology to two cases, one concerning annually observed IMF forecasts for US real GDP growth and one concerning quarterly forecasts for GDP growth in the Netherlands.

Table 4. The EICIE forecasts and realizations

\begin{tabular}{|c|c|c|c|c|}
\hline Quarter & Actuals & EICIE & No-change forecast & Best model forecast \\
\hline 2004Q4 & 1.6 & 1.1 & NA & 1.465293 \\
\hline 2005Q1 & -0.5 & 1.0 & 1.6 & 0.488282 \\
\hline 2005Q2 & 1.3 & -1.5 & -0.5 & 0.587519 \\
\hline 2005Q3 & 1.3 & 1.6 & 1.3 & 1.472545 \\
\hline $2005 Q^{4}$ & 1.6 & 1.8 & 1.3 & 1.665138 \\
\hline 2006Q1 & 2.9 & 2.3 & 1.6 & 2.395027 \\
\hline 2006Q2 & 2.8 & 2.5 & 2.9 & 2.406960 \\
\hline 2006Q3 & 2.7 & 1.9 & 2.8 & 2.190500 \\
\hline 2006Q4 & 2.7 & 2.3 & 2.7 & 2.304697 \\
\hline 2007Q1 & 2.5 & 2.5 & 2.7 & 2.271466 \\
\hline 2007Q2 & 2.6 & 3.1 & 2.5 & 2.487926 \\
\hline 2007Q3 & 4.2 & 2.8 & 2.6 & 3.124916 \\
\hline 2007Q4 & 4.5 & 3.5 & 4.2 & 3.460255 \\
\hline 2008Q1 & 3.3 & 3.5 & 4.5 & 2.918277 \\
\hline 2008Q2 & 3.0 & 2.7 & 3.3 & 2.554389 \\
\hline 2008Q3 & 1.8 & 1.8 & 3.0 & 1.755467 \\
\hline 2008Q4 & -0.6 & 1.4 & 1.8 & 0.557314 \\
\hline 2009Q1 & -4.5 & -1.3 & -0.6 & -1.974944 \\
\hline 2009Q2 & -5.4 & -2.0 & -4.5 & -2.581272 \\
\hline 2009Q3 & -3.7 & -2.6 & -5.4 & -1.984764 \\
\hline 2009Q4 & -2.2 & -1.3 & -3.7 & -0.936152 \\
\hline 2010Q1 & 0.6 & 2.0 & -2.2 & 1.270588 \\
\hline 2010Q2 & 2.2 & 0.6 & 0.6 & 1.593536 \\
\hline 2010Q3 & 1.9 & 1.6 & 2.2 & 1.743534 \\
\hline 2010Q4 & 2.5 & 2.0 & 1.9 & 2.128720 \\
\hline 2011Q1 & 2.8 & 2.0 & 2.5 & 2.264214 \\
\hline 2011Q2 & 1.6 & 3.1 & 2.8 & 2.036277 \\
\hline 2011Q3 & 0.9 & 2.2 & 1.6 & 1.463181 \\
\hline 2011Q4 & -0.6 & 0.9 & 0.9 & 0.414568 \\
\hline 2012Q1 & -1.1 & -0.5 & -0.6 & -0.210945 \\
\hline 2012Q2 & -0.4 & 0.0 & -1.1 & 0.247955 \\
\hline 2012Q3 & -1.5 & -1.0 & -0.4 & -0.534350 \\
\hline 2012Q4 & -1.7 & -0.7 & -1.5 & -0.539032 \\
\hline 2013Q1 & -1.8 & -0.5 & -1.7 & -0.527099 \\
\hline 2013Q2 & -1.7 & 0.4 & -1.8 & -0.224991 \\
\hline 2013Q3 & -0.4 & 1.1 & -1.7 & 0.561996 \\
\hline 2013Q4 & 0.8 & 1.1 & -0.4 & 1.103975 \\
\hline 2014Q1 & 0.0 & 1.0 & 0.8 & 0.714107 \\
\hline 2014Q2 & 1.1 & 0.6 & 0.0 & 1.096723 \\
\hline 2014Q3 & 1.2 & 1.8 & 1.1 & 1.484478 \\
\hline 2014Q4 & 1.4 & 0.1 & 1.2 & 1.089472 \\
\hline 2015Q1 & 2.5 & 1.2 & 1.4 & 1.900326 \\
\hline 2015Q2 & 1.8 & 1.2 & 2.5 & 1.584172 \\
\hline
\end{tabular}

EICIE, Econometric Institute Current Indicator of the Economy. 


\section{ILLUSTRATIONS}

We first present the various relevant parameter estimates, and then turn to an evaluation of the forecast performance.

\subsection{Benchmarks}

Columns 2 and 3 of Table 4 present the data on the Econometric Institute Current Index of the Economy (EICIE) (available from the website of the Erasmus School of Economics) and the second release data from Statistics Netherlands concerning year-to-year GDP growth observed per quarter. The available data range from 2004Q4

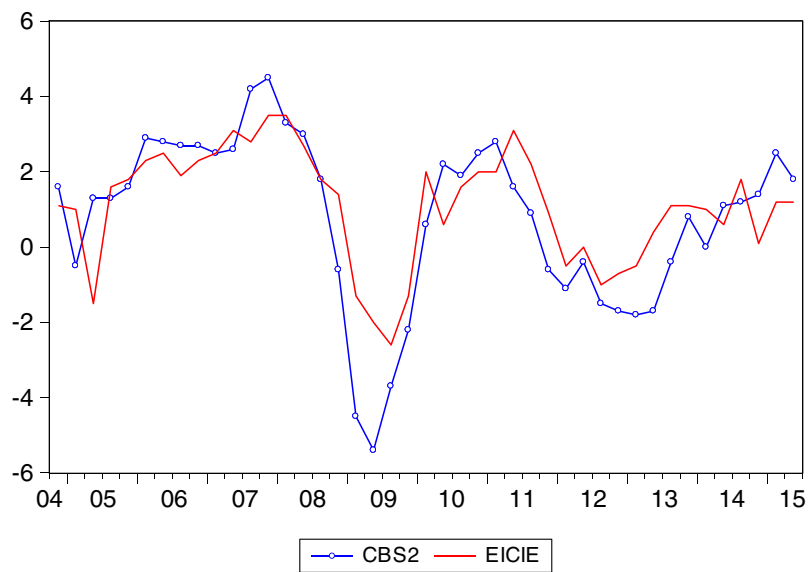

Figure 1. Econometric Institute Current Indicator of the Economy (EICIE) forecasts and actual quarterly GDP growth in the Netherlands (CBS2 concerns the second release data from Statistics Netherlands).

Table 5. The IMF forecasts and realizations

\begin{tabular}{lrrrr}
\hline Year & Actuals & IMF & No-change forecast & Best model forecast \\
\hline 1991 & -0.1 & 1.678600 & NA & 1.660111 \\
1992 & 3.6 & 3.001200 & -0.1 & 2.770674 \\
1993 & 2.7 & 3.141500 & 3.6 & 2.537561 \\
1994 & 4.0 & 2.555300 & 2.7 & 2.843543 \\
1995 & 2.7 & 2.459800 & 4.0 & 2.482932 \\
1996 & 3.8 & 2.022900 & 2.7 & 2.746576 \\
1997 & 4.5 & 2.349000 & 3.8 & 2.962763 \\
1998 & 4.4 & 2.581600 & 4.5 & 2.954253 \\
1999 & 4.7 & 2.030900 & 4.4 & 2.991573 \\
2000 & 4.1 & 2.598900 & 4.7 & 2.874187 \\
2001 & 1.0 & 3.155700 & 4.1 & 2.077139 \\
2002 & 1.8 & 2.189200 & 1.0 & 2.216891 \\
2003 & 2.8 & 2.559400 & 1.8 & 2.518064 \\
2004 & 3.8 & 3.914200 & 2.8 & 2.898140 \\
2005 & 3.3 & 3.540100 & 3.8 & 2.732408 \\
2006 & 2.7 & 3.268400 & 3.3 & 2.547731 \\
2007 & 1.8 & 2.922400 & 2.7 & 2.275648 \\
2008 & -0.3 & 1.939000 & 1.8 & 1.626678 \\
2009 & -2.8 & 0.054956 & -0.3 & 0.796930 \\
2010 & 2.5 & 1.518100 & -2.8 & 2.353165 \\
2011 & 1.6 & 2.312600 & 2.5 & 2.172479 \\
2012 & 2.3 & 1.782300 & 1.6 & 2.320036 \\
2013 & 2.2 & 2.116400 & 2.3 & 2.319659 \\
\hline & & & & \\
\end{tabular}


to 2015Q2. The second release data appear 90 days after the relevant quarter. The EICIE is published during the relevant quarter and hence in fact amounts to a nowcast. Figure 1 gives a graphical impression of the data. An application of ordinary least squares to the regression model as in Equation (3) for the observable data, that is,

$$
y_{t}=\alpha+\beta f_{t}+\varepsilon_{t}
$$

gives $\hat{\alpha}=-0.582$ with standard error 0.229 , and $\hat{\beta}=1.248$ with standard error 0.124 . The Wald test value for the joint hypothesis that $\alpha=0, \beta=1$ is 6.811 , with a $p$ value of 0.033 . This suggests that the EICIE delivers biassed forecasts.

This bias is reinforced by looking at the estimation results for the regression Equation (1), see the second column of Table 6. The estimated $\gamma_{1}$ is quite close to 1 , but the estimated $\gamma_{2}$ is not. The model fit is substantial (0.770), but the Wald test on $\mu=0, \gamma_{1}=1, \gamma_{2}=1$ results in a $p$ value of 0.001 . Hence, on average, the added contribution of the expert, on top of a no-change model forecast, apparently does not improve the final forecast.

The estimated TLS parameters for the regression Equation (3) appear in the left-hand side panel of Table 7. The variance $\sigma_{y}^{2}$ is estimated as 4.865 , the variance $\sigma_{F}^{2}$ is 2.227 , and the covariance between the CBS data (Statistics Netherlands), and the EICIE forecasts is estimated as 2.778. The average observed growth rate is 0.791, and the average nowcast is 1.1. Table 7 reports on the TLS estimates for various values of $\delta$, ranging from 0.7 to 1.3 . Clearly, the estimated parameter values do not change much across this range of $\delta$.

Table 5 presents the IMF forecasts for US real GDP growth for the years 1991 to and including 2013, the columns 2 and 3. Figure 2 gives a graphical impression of the data. The right-hand column of Table 6 shows that final expert forecasts do add something relevant to the no-change forecasts, as the $p$ value of the Wald test on $\mu=0, \gamma_{1}=1, \gamma_{2}=1$ is 0.695 . Moreover, the estimated value of $\gamma_{2}$ is 0.9543 , which is quite close to 1 . So the contribution of the IMF experts is unbiased and relevant. The variance $\sigma_{y}^{2}$ is estimated as 3.024, the variance $\sigma_{F}^{2}$ is 0.610 , and the covariance between the actual data and the IMF forecasts is estimated as 0.781 . The average observed

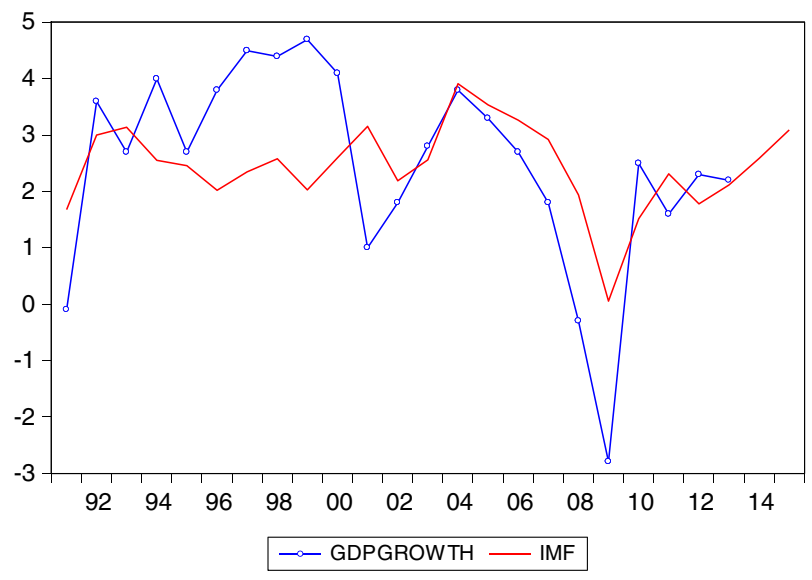

Figure 2. IMF forecasts and actual annual GDP growth rates (in the USA).

Table 6. Regression of actuals on past actuals and differences between judgmental forecasts and past actuals, that is, $y_{t}=\mu$ $+\gamma_{1} y_{t-1}+\gamma_{2}\left(f_{t}-y_{t-1}\right)+\varepsilon_{t}$ and the Wald test on $\mu=0, \gamma_{1}=1, \gamma_{2}=1$

\begin{tabular}{lcr}
\hline Parameter & EICIE & \multicolumn{1}{c}{ IMF } \\
\hline$\mu$ & $-0.329(0.230)$ & $-0.236(1.040)$ \\
$\gamma_{1}$ & $1.135(0.120)$ & $1.152(0.404)$ \\
$\gamma_{2}$ & $0.691(0.215)$ & $0.943(0.469)$ \\
$R^{2}$ & 0.770 & 0.338 \\
$P$ value of Wald test & 0.001 & 0.695 \\
\hline
\end{tabular}

EICIE, Econometric Institute Current Indicator of the Economy. 
PHILIP HANS FRANSES AND BERT DE BRUIJN

Table 7. TLS parameter estimates for various values of $\delta$

\begin{tabular}{lllll}
\hline & \multicolumn{2}{c}{ EICIE } & \multicolumn{2}{c}{ IMF } \\
\cline { 2 - 4 }$\delta$ & $\hat{\alpha}_{T L S}$ & $\hat{\beta}_{T L S}$ & $\hat{\alpha}_{T L S}$ & $\hat{\beta}_{T L S}$ \\
\hline 0.7 & -0.993 & 1.622 & -6.053 & 3.526 \\
0.8 & -0.977 & 1.608 & -5.940 & 3.479 \\
0.9 & -0.963 & 1.594 & -5.828 & 3.433 \\
1.0 & -0.949 & 1.582 & -5.719 & 3.388 \\
1.1 & -0.936 & 1.570 & -5.610 & 3.343 \\
1.2 & -0.924 & 1.559 & -5.506 & 3.300 \\
1.3 & -0.912 & 1.549 & -5.405 & 3.258 \\
\hline
\end{tabular}

EICIE, Econometric Institute Current Indicator of the Economy.

Table 8. Forecast performance

\begin{tabular}{lccc}
\hline & Judgement & No-change & Best model \\
\hline EICIE & $21 \%$ & $35 \%$ & $44 \%$ \\
IMF & $27 \%$ & $23 \%$ & $50 \%$ \\
\hline
\end{tabular}

Fraction that forecasts have the lowest absolute forecast error across three forecasts, that is, the judgmentally adjusted forecast, the no-change forecast and the best model forecast.

EICIE, Econometric Institute Current Indicator of the Economy.

Table 9. Quarters and years in which the final forecasts improve on both the no-change forecast and the best model forecast

\begin{tabular}{lll}
\hline EICIE & Quarters & 2007Q1, 2008Q1, 2008Q2, 2008Q3, 2009Q3, 2009Q4, 2012Q2, 2012Q3, 2013Q4 \\
IMF & Years & $1992,2002,2003,2004,2005,2013$ \\
\hline
\end{tabular}

EICIE, Econometric Institute Current Indicator of the Economy.

growth rate is 2.483 , and the average forecast is 2.421 . Table 7 reports on the TLS estimates for various values of $\delta$, ranging from 0.7 to 1.3 , and again the estimated parameter values do not change much across this range of $\delta$.

\subsection{Forecast performance}

For further analysis, we now set $\delta=1$. Table 8 presents the fraction of times that forecasts have the lowest absolute forecast error across three forecasts, that is, the final judgmentally adjusted forecast, the no-change forecast and the best-model forecast. As could be expected, and by creation, the best-model forecast is best in about half the cases across these sets of forecasts. The no-change forecast seems on average about equally good as the final expert forecast. But still, in 1 of 4 quarters or years, the expert touch does seem to improve on both benchmark forecasts.

Table 9 zooms in on the quarters and years where the expert forecasts were more accurate than the benchmarks. Clearly, the quarters 2009Q3, 2009Q4, 2012Q2 and 2012Q3 as well as the years 2002-2005 and 2013 are recovery quarters and years. So it seems that the expert adjustment was most useful in these recovery periods. Apparently, econometric models can need the help of experts, particularly in these business cycle episodes.

\section{CONCLUSION}

We have proposed a simple methodology to benchmark final macroeconomic forecasts. This is necessary as those final forecasts are typically the combination of an econometric model-based forecast and a manual modification by an expert. The analyst usually does not know the specific weights in the combination. Illustrations to two sets of GDP growth forecasts showed the merits of the methodology. 


\section{REFERENCES}

Carroll RJ, Ruppert D. 1996. The use and misuse of orthogonal regression in linear errors-in-variables models. The American Statistician 50: 1-6.

Deming WE. 1943. Statistical Adjustment of Data, Wiley: London.

Franses PH. 2014. Expert Adjustments of Model Forecasts; Theory, Practice and Strategies for Improvement, Cambridge University Press: Cambridge.

Franses PH, Kranendonk H, Lanser D. 2011. One model and various experts: evaluating Dutch macroeconomic forecasts. International Journal of Forecasting 27: 482-495.

Franses PH, Maassen N. 2015. Consensus Forecasters: How Good are They Individually and Why?, Econometric Institute Report 2015-21, Erasmus University: Rotterdam.

Fuller WA. 1987. Measurement Error Models, Wiley: New York.

Koopmans T. 1937. Linear Regression Analysis of Economic Time Series, De Erven F. Bohn NV: Haarlem the Netherlands.

Linnet K. 1990. Estimation of the linear relationship between the measurements of two methods with proportional errors. Statistics in Medicine 9: 1463-1473.

Vuchelen J, Gutierrez M-I. 2005. A direct test of the information content of the OECD growth forecasts. International Journal of Forecasting 21: 103-117.

Wansbeek T, Meijer E. 2000. Measurement Error and Latent Variables in Econometrics, North-Holland: Amsterdam. 